\title{
Thematic Progression in the Academic Writing of Non-English Major Postgraduates in Mainland China
}

\author{
Wenna Dou ${ }^{1, ~ *, ~ H u ~ Z h a o ~}{ }^{2}$ \\ ${ }^{1}$ Department of Foreign Languages, School of Human and Law, Beijing University of Civil Engineering and Architecture, Beijing, China \\ ${ }^{2}$ School of Environment and Energy Engineering, Beijing University of Civil Engineering and Architecture, Beijing, China
}

Email address:

douwenna@bucea.edu.cn (Wenna Dou), 1542366883@qq.com (Hu Zhao)

${ }^{*}$ Corresponding author

To cite this article:

Wenna Dou, Hu Zhao. Thematic Progression in the Academic Writing of Non-English Major Postgraduates in Mainland China. International Journal of Language and Linguistics. Vol. 7, No. 1, 2019, pp. 1-7. doi: 10.11648/j.ijl1.20190701.11

Received: December 12, 2018; Accepted: December 25, 2018; Published: January 21, 2019

\begin{abstract}
Through combing out eight patterns of Thematic Progression which are most frequently used in English scientific and technological academic papers, this study aims to examine the usage tendency and internal influencing factors of Thematic Progression patterns in English research abstracts written by postgraduates majoring in architecture and civil engineering in mainland China. Combining corpus-based analyses and group-focus interviews, results show that the overall writing level of Chinese non-English major postgraduates could reach the publishing standards of international journals in the facet of the number of words and sentences. Especially, "Simple Linear Thematic Progression" and "Thematic Progression with a Constant Theme" were the most frequently used in the students' abstracts. Findings suggest that the tendency of using "Simple Linear Thematic Progression" was driven by the logic of students' thinking in the academic writing process, while the application of "Thematic Progression with a Constant Theme" was correlate with genre types. The specific Move Structure in research abstracts was also found to be potentially affecting the usage tendency of special Thematic Progression patterns. Both theoretical and practical implications were discussed in this study.
\end{abstract}

Keywords: Thematic Progression, Academic Writing, Non-English Major Postgraduates, Corpus-Based Analyses, Case Study

\section{Introduction}

Theme-Rheme development is of great significance for understanding the semantic cohesion and textual connotation at the micro-level of discourses. Compared with the traditional grammar which emphasizing the linguistic forms of discourses, the study of Theme-Rheme development pays more attention to the importance of the content and meaning of discourses at the macro levelsentence function. Extant studies have shown that thematic-rheme relationship has been studies in various domains including news reports, advertisements and academic writing[1-3]. Among the above, academic writing gradually arouses scholars' attention, because it has become an inevitable ability for postgraduates to master, especially in China. In some disciplines, Chinese postgraduates are required to publish research papers in English so that they can graduate successfully. Architecture and Civil Engineering is one of them.

However, domestic and foreign scholars mostly focus on academic writing ability of either students whose English is their mother tongue or English major students[4-6], ignoring the problems of L2 learners, especially non-English major students. Moreover, there are rare studies examining the research abstracts written by non-English major postgraduates in mainland China. Therefore, this study aims at exploring the linguistic features via Self-Compiled Architecture and Civil Engineering English Learner Corpus as a case study. Specifically, this study investigates the internal influencing factors of the patterns of Thematic Progression with the method of focus-group interviews. 


\section{The Classification of Thematic Progression}

Mathesius, the founder of the Prague School, first proposed the concept "Sentence View" [7]. In the subsequent studies, he pointed out that the actual division of a sentence should be made by semantics and then the concepts of "Theme" and "Rheme" in the sentence were put forward. In his opinion, "Theme" was the object of a statement, which corresponded to the old information while "Rheme" was based on the expansion of topic corresponding to the new information. Subsequently, linguist Frantisek Danes proposed a different explanation. He defined "Theme" as " the words expressed by the starting point", while "Rheme" as "the words used to demonstrate the core". He claimed that this division of "Theme" and "Rheme" was determined by discourse contents and contexts. His notion was completely distinguished from that of previous studies, which was conducted from the grammatical perspective focusing on the grammatical form such as the Subject and the Object.

In 1974, linguist Frantisek Danes[8] first proposed the "Thematic Progression(TP)" in the article "Functional sentence perspective and the organization of the text". Based on the study of Czech, German and English scientific discourses, he analyzed the complex sentence structure in these discourses and found out that the "connexity" of discourse was mainly achieved through Thematic Progression. Furthermore, he advanced five main patterns of Thematic Progression.

\subsection{Simple Linear Thematic Progression}

In this pattern, Rheme in the first clause is the Theme of the second clause. Likewise, Rheme in the second clause is the Theme of third clause. The old and new information contained in the discourse is alternated by a linear pattern so that the intrinsic connectivity of sentences can be achieved. The specific expressions are shown as follows: (T stands for Theme, R stands for Rheme, and numbers mean the levels of sentence meaning).

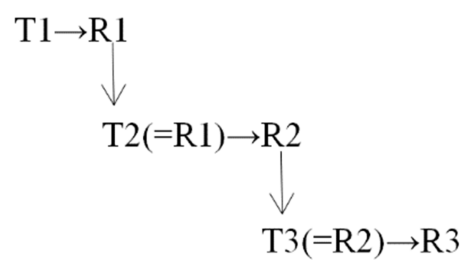

Figure 1. Simple Linear Thematic Progression.

Example $^{1}$ : A composite solid alkaline activator (T1) / was incorporated into the slag-red mud mixed system in order to develop an alkali slag-red mud cement with excellent performance (R1). The cement (T2=R1) / has good stability, when the ratio of slag and red mud in cement was 70:30 (R2). The ratio $(\mathrm{T} 3=\mathrm{R} 2) /$ will be retested in this research(R3).

\subsection{Thematic Progression with A Constant Theme}

As for this pattern, each clause has same Theme, but different Rhemes (R1, R2, R3...Rn). Different Rhemes are connected to each other through same Theme to constitute semantic intrinsic cohesion within a piece of discourse. It is illustrated in Figure 2:

$$
\begin{aligned}
& \mathrm{T} 1 \rightarrow \mathrm{R} 1 \\
& \mathrm{~T} 1 \rightarrow \mathrm{R} 2 \\
& \mathrm{~T} 1 \rightarrow \mathrm{R} 3
\end{aligned}
$$

Figure 2. Thematic Progression with A Constant Theme.

Example: The cold-formed thin-walled steel structure (T1) / is a new type of structure system (R1). It (T1) / has been developed rapidly in the world and widely used in the residential structures in recent twenty years (R2). But this kind of steel structure (T1) / is still in the initial stage of research and application in our country (R3).

\subsection{Thematic Progression with Derived Themes}

As for this pattern, the Theme of the first clause acts as "hyper-theme", $\mathrm{T}$ is the Superordinate, while T', T", T"” are the Hyponyms, which lead to the following different Rhemes respectively. In this way, new and old information can be transmitted and connected. The specific expressions are shown as follows:

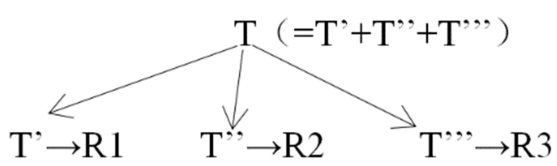

Figure 3. Thematic Progression with derived Themes.

Example: Many research(T) / have shown that the waste vegetable oil can be used for asphalt regeneration (R). Some studies ( $\left.\mathrm{T}^{\prime}\right)$ / have verified the road performance of recycled asphalt mixture (R1). Other studies (T") / have found that vegetable oil can effectively improve the physical, chemical properties of aged bitumen (R2). However, little research ( $\mathrm{T}^{\prime \prime}$ ) / have revealed the relationship between the aging behavior of recovered bitumen and vegetable oil re generator(R3).

\subsection{Thematic Progression with Derived Rhemes}

Similar to "Thematic Progression with Derived Themes", in this pattern, the Rheme in the first clause is the Superordinate, while the Theme of the second clause is derived from the Rheme of the first clause, known as R'. The Theme of the third clause is also derived from the Rheme of the first clause, known as R". By analogy, the transmission of discourse information is realized. In the way, the Rheme of the first clause is a collection of R' R" and R"'. It is illustrated in Figure 4: 


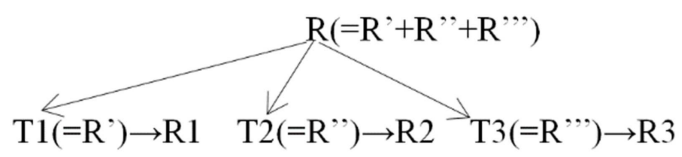

Figure 4. Thematic Progression with Derived Rhemes.

Example: This article (T) / summarizes the scholars' research on the properties of frozen soil from three aspects (R). The first one (T1=R') / is that scholars have studied the relationship between temperature, water content, confining pressure, shear rate, and number of freeze-thaw cycles for permafrost under static loading, using tree-axial shear tests of frozen soils ( $\mathrm{R} 1)$. The second $(\mathrm{T} 2=\mathrm{R}$ ') / is to study the properties of roads and railway sub-grades under dynamic loads of frozen soil (R2). The last aspect (T3=R",')/ is that the researchers combined the macroscopic and microscopic views to study the mechanism of the strength change of frozen soil by analyzing the internal particle contact relationship, the connection form, and the particle state (R3).

\subsection{Skipping Thematic Progression}

As for this pattern, the connection between clauses is disjointed, which needs to be inferred through the whole contextual structure. The Theme in the first clause reappears in the thematic position in other clauses, which are far from the the first clause. The specific expressions are shown as follows:

$$
\begin{aligned}
& \mathrm{Tn} 1 \rightarrow \mathrm{R} 1 \\
& \mathrm{Tn}(=\mathrm{T} 1) \rightarrow \mathrm{Rn}
\end{aligned}
$$

Figure 5. Skipping Thematic Progression.

Example: "Resilient Prefabricated Pre-stressed Steel Frame" with steel plate shear wall (RPPSF-SW) (T1) / is a new structural system that combines lateral stiffness of conventional shear wall and beam-column connection with self-centering capacity(R1) .... Additionally, the RPPSF-SW $(\mathrm{Tn}=\mathrm{T} 1) /$ has relatively favorable ductility and self-centering capacity as well as possessing outstanding energy dissipating capacity $(\mathrm{Rn})$.

Danes believed that Thematic Progression was governed by the purpose of the discourse, and in most cases, it can be interpreted from various angles apart from linguistic perspective. Therefore, based on the above-mentioned Thematic Progression patterns, other patterns could be derived from them. Domestic scholars in China further proposed different syntactic development patterns. Huang Y. proposed that there were seven patterns, Huang, G. W. six kinds and $\mathrm{Hu}, \mathrm{Z}$. L. four patterns [9-12].

Combining all the related research in China and abroad, this paper combs out three more Thematic Progression patterns commonly employed in English scientific articles, namely, Intersection Thematic Progression, Inductive Thematic Progression and Centralized Thematic Progression.

\subsection{Intersection Thematic Progression}

In the pattern, the Rheme in the second clause is the Theme of first clause, while the Rheme in the third clause is the Theme of second clause, and the rest can be done in the same manner. It is illustrated in Figure 6:

$$
\begin{aligned}
& \mathrm{T}_{1} \rightarrow \mathrm{R}_{1} \\
& \mathrm{~T}_{2} \rightarrow \mathrm{R}_{2}\left(=\mathrm{T}_{1}\right) \\
& \cdots \\
& \mathrm{T}_{\mathrm{n}} \rightarrow \mathrm{R}_{\mathrm{n}}\left(=\mathrm{T}_{\mathrm{n}-1}\right)
\end{aligned}
$$

Figure 6. Intersection Thematic Progression.

Example: Nowadays, the green building materials (T1) /occupies the main theme of architectural industry(R1). Therefore, people(T2) / pay more attention to the promotion and application of green building materials in field of construction $(\mathrm{R} 2=\mathrm{T} 1)$.

\subsection{Inductive Thematic Progression}

This pattern shows that the combination of Theme and the Rheme within a clause becomes a new Theme in the following clause. In other words, the Theme of the latter clause is the comprehensive expression of the Theme and the Rheme in the preceding clause or those above. The specific expressions are shown as follows:

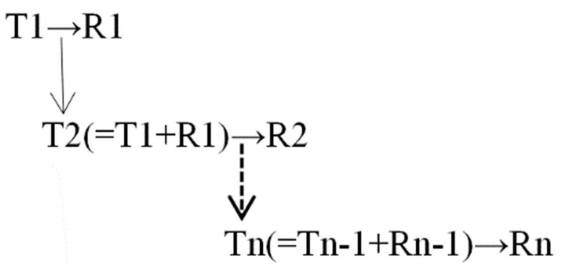

Figure 7. Inductive Thematic Progression.

Example: Numerical models(T1) / are compared with experiment measurements in terms of displacement, acceleration and amplification factor of the structural model and the soil(R1). The comparison (T1) / shows that the numerical results match the experimental measurements quite well(R2). All the above results show $(\mathrm{T} 3=\mathrm{T} 1+\mathrm{T} 2) /$ that the models are valid and can be adopted for further analysis(R3).

\subsection{Centralized Thematic Progression}

As for this pattern, each clause has a different Theme but the same Rheme. It is illustrated in Figure 8:

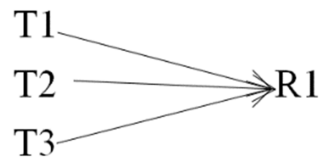

Figure 8. Centralized Thematic Progression.

Example: Experimental research and numerical simulations(T1) / were conducted to investigate the mechanical performance of PSCC(R1). The coefficient of 
friction and the pre-load of high-strength bolts (T2) / were used to explore initial stiffness, decompression moment, gap-opening stiffness, imminent moment of gap-opening, maximum gap-opening moment, cable forces of the steel strands of PSCC (R1). Therefore, parametric analysis (T3) /can lay the foundation for further study of PSCC's performance (R1).

Based on the above-mentioned eight Thematic Progression patterns most commonly used in the scientific papers, this study aims at analyzing the usage tendency of the Thematic Progression patterns by employing the statistical analysis software SPSS. On the other hand, With the help of NVivo qualitative analysis software, the follow-up interviews are further conducted in order to explore the relevant factors that may affect the application of the Thematic Progression patterns. The specific research questions are shown as follows:

(1) Which Thematic Progression patterns are frequently used in the non-English major postgraduates' research abstracts?

(2) What's the reason behind the above phenomenon from students' angle?

(3) What are the possible internal factors that can influence the application of Thematic Progression patterns in the students' research abstracts?

\section{Research Design}

\subsection{Data Collection}

The procedure of data collection follows three principles. Firstly, those articles were written by postgraduates majoring in architecture and civil engineering. Secondly, all the articles were written in English. Thirdly, the articles were collected after all the students had taken a Academic English Writing Course for one semester. At the end of the semester, there are 120 articles, including 59 papers on construction engineering and 61 papers on civil engineering.

\subsection{Data Processing}

At the beginning, the collected articles were converted into to text format in order to unify the format and facilitate information annotation and retrieval in the corpus, for there were two main formats of English papers submitted by students-word format and pdf format. Papers in word format were saved by using ANSI encoding. Papers in PDF format are converted to text format (.txt) through Solid PDF Tools v9. All the data were stored on a mobile hard disk for subsequent loading and updating.

Moreover, the sample of raw data were chosen at random in order to conduct a pilot analysis. Although the formats of raw data were processed, there were still some problem, such as letters, punctuation, and so on. So the raw data were cleaned by employing software Text Organizer5.0.

Finally, Architecture and Civil Engineering English Learner Corpus was built after the following three stepsmeta-information labeling, word segmentation and part-of-speech tagging.

\subsection{Data Analysis}

At first, 50 abstracts of academic papers were randomly selected from Architecture and Civil Engineering English Learner Corpus. There were totally 50 paragraphs and the number of sentences and words in each sentence was counted. Based on the above data, this study carried out a descriptive statistical analysis by employing SPSS 19.0.

What's more, Themes and Rhemes of the selected 50 English abstracts were labeled respectively with the help of NVivo 11 in order to analyze the logical relationship of each paragraph. The results were demonstrated through the frequency of eight Thematic Progression patterns appearing in abstracts of papers.

And then, according to the statistical results, this study selected 16 graduate students for interviews. Among them, every two students used same Thematic Progression patterns. Based on usage frequency of the Thematic Progression, the study investigated the tendency in application.

At last, this study analyzed the influencing factors of the Thematic Progressions in research abstracts from the perspective of genre analysis.

\section{Results and Discussion}

\subsection{The Overall Writing Status of Research Abstracts of the Non-English Major Postgraduates in China}

This study conducted a descriptive statistical analysis of the collected data of 50 research abstracts of academic papers. The results are shown in table 1 .

Table 1. Descriptive statistical analysis.

\begin{tabular}{|c|c|c|c|c|c|c|}
\hline & $\mathbf{N}$ & Minimum & Maximum & $\mathbf{M}$ & SD & Total \\
\hline Sentences Number & 50 & 3 & 12 & 6.34 & 2.047 & 317 \\
\hline Word Number & 50 & 74 & 344 & 165.22 & 48.627 & 8261 \\
\hline
\end{tabular}

As shown in Table 1, there are 50 paragraphs altogether, 317 sentences, and 8261 words. The average sentence number is 6.34 and The average word number in each paragraph is 165.22 . The results of this study are consistent with the research findings of Cao, Z. H. and Li, L. [3]. They analyzed 50 abstracts of scientific articles published in the journal of Nature. Their research findings showed that the average sentence number is 6.76 and the average word number in each paragraph is 162 .

Hence, the above comparison has shown that from the aspects of the number of words and sentences in the abstracts, Chinese non-English majors postgraduates master the basic requirements for publishing papers in top international academic journals. 
However, by examining the specific cases, this study finds that there are still some problems. In terms of the number of sentences, there are 24 abstracts which contain 6 or 7 sentences , accounting for $48 \%$ of the total; 10 abstracts are composed of 3 or 4 sentences, accounting for $20 \%$ of the total; 5 abstracts are made up of between 10 and 12 sentences, accounting for $10 \%$ of the total.

Through careful analysis of two abstracts with the fewest sentences, the findings show that the writers tends to use simple conjunctions to combine several simple sentences into one complex sentence. This is shown in the sixth sentence of the 30th abstracts as follows:

"The results show that the reinforcement effect of the reinforcement system proposed in this paper is obvious, and the ultimate bearing capacity of the strengthened square ditch cover plate is increased by $28 \%$ at maximum, and the mid-span deflection is decreased by $25 \%$."(030-06).

As shown above, the writer combines three research results with two "and"s. Composed in this way, the abstract not only lacks variety of sentence complexity, but also creates inconvenience for readers to understand the main content. This phenomenon suggests that students need more effort to learning the rules of composing complex sentence structure. Furthermore, as for the language teachers, they should pay more attention to this kind of language problem during the teaching process in order to enhance the students' writing ability effectively.

Similarly, the study shows that there is a common feature, through examining the two abstracts with the most sentences. The writers of these two abstracts do not have a good grasp of the Five Core Elements for writing a paper abstract proposed by Santos,M., namely: research background, research content, research methods, research findings, and discussion [13]. As a result, it leads to lengthy abstracts without highlighting the core elements.

\subsection{Tendency of Using Specific Thematic Progression Patterns in Research Abstracts of Academic Writing}

With the aid of qualitative analysis software NVivo 11, this study tags Themes and Rhemes used in the abstracts of scientific papers sentence by sentence. This task is done by two English teachers whose major were both linguistics. If they have different divisions, those controversial sentences will be re-tagged by third person.

The eight Thematic Progression patterns were tagged paragraph by paragraph based on the above principle. The usage frequency of eight Thematic Progression patterns are shown as Table 2 .

Table 2. Usage frequency of eight Thematic Progression patterns.

\begin{tabular}{lll}
\hline Types & Usage Frequency & Percentage(\%) \\
\hline Simple Linear Thematic Progression & 27 & 23.68 \\
Thematic Progression with A Constant Theme & 20 & 17.54 \\
Thematic Progression with Derived Themes & 19 & 16.66 \\
Thematic Progression with Derived Rhemes & 18 & 15.79 \\
Skipping Thematic Progression & 10 & 8.77 \\
Intersection Thematic Progression & 9 & 7.89 \\
Inductive Thematic Progression & 7 & 6.14 \\
Centralized Thematic Progression & 4 & 3.50 \\
Total & 114 & 100 \\
\hline
\end{tabular}

Table 2 shows that "Simple Linear Thematic Progression" and "Thematic Progression with A Constant Theme" are most frequently used among eight patterns, which together accounts for $41.22 \%$ of the total. Previous research on the Thematic Progressions of scientific papers published in the international journals also show that the most popular patterns employed by scholars are "Simple Linear Thematic Progression" and "Thematic Progression with A Constant Theme" [3]. Therefore, the analysis shows that, from the perspective of discourse structure, the writing ability of Chinese postgraduates majoring in architecture and civil engineering meets the basic requirements of international research papers' writing in English.

Through face-to-face interviews with two writers using "Simple Linear Thematic Progression" in the abstracts of their articles, it demonstrates that the tendency of using Simple Linear Thematic Progression is driven by the logic of people's thinking in the academic writing process.

Obviously, it is easier for people to understand old information than new one. It takes less effort for people to acquire old information than new information. This logic is realized by "Simple Linear Thematic Progression". Within a discourse, the old information presents first, and the new information follows up. The old information paves the way for the new information in advance. In this way, the transmission from old to new information is achieved smoothly, which leads to the connection of the internal structure of a discourse. Thus, "Simple Linear Thematic Progression" is in line with people's logic of thinking in the writing process. It establishes coordination by tacit agreement between authors and reader, so that the communication between them is more effective.

In the same way, based on the interviews conducted for the other two writers using "Thematic Progression with A Constant Theme" in the abstracts of their articles, it shows that the application of "Thematic Progression with a Constant Theme" is correlate with genre types. Among the 50 abstracts selected from the Architecture and Civil Engineering English Learner Corpus, only one of them is a literature review, and the rest are all experimental studies.

Evidently, experimental research papers mainly focus on a specific problem found in the construction process of 
Architecture and Civil Engineering and then verify the possible solutions through various methods of experiments. The key concept emerging from this problem is not only the key word of the research paper, but also the key word in research abstract. Based on this key concept, different writers explore it from multiple dimensions.

Therefore, the results suggest that the key word plays the role of Theme in a clause from semantic perspective. In this way, it leads to a tendency for writers majoring in Architecture and Civil Engineering to use "Thematic Progression with A Constant Theme" in their research abstracts where the Theme of a clause is the leading role in the whole discourse content.

\section{3. “Move Structure” as the Internal Influencing Factor}

Different from the traditional discourse analysis which focusing on the micro structure of language, Genre Analysis emphasizes the importance of the macro structure of discourses. Each type of genre generated from a specific structure of a discourse is a symbolic system [14]. Research abstracts of academic writing of non-English major postgraduates in mainland China is a specific discourse, and its internal structure is embodied through specific Moves[15]. From the perspective of textual cohesion, the specific Move structure is centered on the communicative purpose, thus it can realize the inner connection of a discourse content at the sentence or paragraph level.

This study carries out a carefully analysis of 50 research abstracts of students by employing John M. Swales' genre analysis theory as the research framework. The results shows that the structure of students' research abstracts is mainly composed of three major Moves, namely, Move1 which refers to searching for the "blanks" or gap in previous research, Move2 which refers to filling up the gap and Move3 which refers to the statements of research results .

Through In-depth analysis of the relationship between the clauses of each Move, the findings of this study demonstrate two features. On the one hand, there is a certain regularity in the usage tendency of Thematic Progression, which means writers tend to use specific Thematic Progression patterns in different Moves. On the other hand, this regularity is determined by the communicative purposes conveyed by each Move. See Table 3 for details:

Table 3. The Usage of Thematic Progression in Each Move.

\begin{tabular}{lll}
\hline Moves & Thematic Progression used frequently & Communicative Purpose \\
\hline Move 1 & $\begin{array}{l}\text { Simple Linear Thematic Progression } \\
\text { Thematic Progression with A Constant Theme }\end{array}$ & Summarizing previous studies and find out research problems \\
Move 2 & $\begin{array}{l}\text { Simple Linear Thematic Progression } \\
\text { Intersection Thematic Progression } \\
\text { Inductive Thematic Progression } \\
\text { Skipping Thematic Progression }\end{array}$ & Expatiating on the implementation process of research and problem solving \\
\hline
\end{tabular}

As shown in Table 3, the discourse structure of research abstracts consists of three Moves, each of which corresponds to a specific Thematic Progression. In Move 1, the writers mainly summarize and analyze previous research in the relevant fields in order to find out research gaps, which lay a practical foundation to put forward their own research questions. In this Move, the writers usually use "Simple Linear Thematic Progression" and "Thematic Progression with A Constant Theme". The selection tendencies of these two models are derived from the logic of thinking and the genre of the thesis.

In Move 2, the writer's main purpose is to explain the specific procedure of their own research, including research objects, research methods, research tools and so on. In this Move, the writers frequently use "Simple Linear Thematic Progression" and "Intersection Thematic Progression". The usage tendency of the latter one is mainly determined by the grammatical sentence structure of the abstracts. Passive voice is the most dominant one. In the collected data, "Intersection Thematic Progression" pattern appears 18 times, of which the passive voice appeares 14 times, accounting for $77.78 \%$ of the total, as shown in the following example:

"A number of methods of integrating Geo-technical data into the BIM process (T1) / were considered and critically reviewed(R1). The research(T2) / was carried out using a hybrid approach combining empirical and explanatory strategies(R2=T1)."

Combined with the data from the interviewing those writers who used passive voice in their research abstracts, it shows that students are accustomed to translating Chinese into English directly in the process of writing, which is greatly influenced by Chinese language logic. Therefore this study suggests that the passive voice is overused in students' research abstracts, which is result from the writers' applying Chinese way of thinking to the process of writing in English. When analyzing this part, most typical structures of passive voice are shown as follows:

(1) "in this study, themethod was used."

(2) "data is analyzed."

(3) "structural model is built."

In Move 3, the writers' main communicative purpose is to present the research findings to the readers. In this Move, the writers usually use "Inductive Thematic Progression" and "Skipping Thematic Progression". The usage tendency of the former is determined by writers' communicative purpose, namely: summary. Differently, the usage tendency of the latter stems from the writers' reinterpretation of the key concepts of their research at this time, which echoes to research questions of their studies.

In summary, the Move structure based on 
communicative function and purpose is an intrinsic factor that affects the use of Thematic Progression in research abstracts. The study suggests that second language teachers should not only focus on characteristics of writing at the sentence-grammatical level, but also pay attention to the communicative features at the functional discourse level.

\section{Conclusion}

This article focuses on Thematic Progression in the research abstracts of postgraduate students majoring in architecture and civil engineering in mainland China. It combs out eight Thematic Progression patterns which are most frequently used in academic writing. Moreover, Based on the quantitative method, it analyzes the usage tendency of Thematic Progression patterns. What's more, through in-depth interviews, this study finds out Move structure of research abstracts plays an role in affecting students' employing the Thematic Progression in their research abstracts.

This study contributes to the body of literature on the Thematic Progression in several important ways. On the one hand, this study is among the first to examine the research abstracts of English learners in mainland China. It provides not only experimental data to identity the real writing problems of English learners, but also teaching aids for language teachers to apply in the teaching process. On the other hand, the findings from this study will help to expand the research scale of language teachers which aims at effectively improving students' L2 academic writing ability in China.

This is also the first study to examine the research abstracts in the field of architecture and civil engineering in mainland China. This offers a different context from most previous Thematic Progression studies. Findings from this study can demonstrate the special linguistic features for postgraduates to master when they compose academic papers in English in order to graduate successfully in mainland China. Therefore, this study provides a worthwhile assessment of Thematic Progression on different cultural context. Because of the limitations of data collection, more research can include different academic research fields in order to explore the regularities of writing characteristics based on different majors in the future.

\section{Acknowledgements}

This article is supported by "The Fundamental Research Funds for Beijing University of Civil Engineering and Architecture" (No. X18220)

\section{References}

[1] Seyyedeh, N.and Esmaeel, N. (2017). Thematic structure and translation: A case study of the translation of English news into Persian, Lingua, 194, 26-50.

[2] Fang, L. and David, K. (2018). A science for verbal art: Elizabeth Gaskell's contribution to a critique of political economy, Language Sciences, 70, 92-102.

[3] Cao, Z. H. And Li, L. (2015). Analysis of the Thematic Progression in the Abstracts of Nature's Articles. Journal of Xidian University(Social Science Edition), 25-6, 104-111.

[4] Derya A.and Yasemin, B.(2019). An exploratory study on factors influencing undergraduate students' academic writing practices in Turkey, Journal of English for Academic Purposes, 37, 88-103.

[5] Xu, F. (2016). Coherence Modeling for English Student Essay Based on Theme-rheme Structure Theory. Journal of Chinese Information Processing, 1, 115-123.

[6] Feng, Y. T. (2017). A Study on Cognitive Thinking of Thematic Progression Patterns in English Thesis Writing for Application-Oriented University English Major Students. The Guide of Science \& Education, 11, 48-50.

[7] Mathesius, V. (1929). Zur Satzperspektive in modern English. Herrigs Archiv, 155, 202-210.

[8] Danes, F. (1974). Functional sentence on the semantic structure of the sentence. In F. Danes (ed.). Papers on the Functional Sentence Perspective. Prague: Academia, 106-128.

[9] Huang, Y. (1985). On Theme and Rheme in English. Journal of Foreign Languages, 5, 32-36.

[10] Huang, G. W. (2001). Theory and Practice of Discourse Analysis. Shanghai: Shanghai Foreign Language Education Press.

[11] Hu, Z. L. (1994). Cohesion and Coherence of Text. Shanghai: Shanghai Foreign Language Education Press.

[12] Liu, D. (2012). English-Chinese Thematic Structure Contrast and English Writing Teaching Method Study. Foreign Language Research, 3,121-125.

[13] Santos, M. (1996). The textual organization of research paper abstracts in applied linguistic. Text-Interdisciplinary Journal for the Study of Discourse, 4, 481-500.

[14] Bhatia, V. K. (1993). Analysing Genre: Language Use in Professional Settings. New York: Longman Group UK Limited.

[15] Swales, J. (1990). Genre Analysis: English in Academic and Research. Cambridge: Cambridge University Press. 